\title{
Awareness of Intravenous Contrast Media Effects on Renal Function in Saudi Arabia- An Observational Study
}

\author{
Rani Ahmad', Rahaf Almoallim², Duaa Basalem, Nashwa Helabi ${ }^{4}$ Shahad Aleiidi ${ }^{5}$, Fatemah Albugmi ${ }^{6}$ \\ 1Department of Radiology, Faculty of Medicine, King Abdulaziz University Hospital, Jeddah, Saudi Arabia. \\ 2Department of Radiology, Faculty of Medicine, King Abdulaziz University Hospital, Jeddah, Saudi Arabia. \\ ${ }^{3}$ Department of Radiology, Faculty of Medicine, King Abdulaziz University Hospital, Jeddah, Saudi Arabia. \\ ${ }^{4}$ Department of Radiology, Faculty of Medicine, King Abdulaziz University Hospital, Jeddah, Saudi Arabia. \\ ${ }^{5}$ Department of Radiology, Faculty of Medicine, King Abdulaziz University Hospital, Jeddah, Saudi Arabia. \\ ${ }^{6}$ Department of Radiology, Faculty of Medicine, King Abdulaziz University Hospital, Jeddah, Saudi Arabia.
}

\section{ABSTRACT}

\section{BACKGROUND}

Intravenous contrast media (IVCM) are routinely used in different imaging techniques, such as computed tomography (CT) and magnetic resonance imaging (MRI), but can cause adverse effects on renal function. This study aims to evaluate the level of awareness of IVCM and their effects on renal function among members of the general public visiting King Abdulaziz University Hospital (KAUH) in Jeddah, Saudi Arabia.

\section{METHODS}

An observational cross-sectional study design was used. It was conducted in September 2018 on participants aged $\geq 18$ years who completed an electronic selfadministered questionnaire to assess their knowledge of the adverse renal effects of IVCM. Data was analyzed using descriptive and inferential statistics. Chi-squared test was used to evaluate the level of knowledge among residents regarding the possible adverse renal events that can be caused by IVCM.

\section{RESULTS}

Most participants (56.8\%) had no knowledge of IVCM and were unable to recognize the risk factors associated with IVCM and their potential impact on the kidneys. Social media was the most chosen source of information among those who had variable degrees of awareness. A statistically significant association was found between the educational level and degree of knowledge about the importance, application, and adverse events of IVCM. Most of the respondents lacked knowledge of the risk factors associated with contrast-induced nephropathy.

\section{CONCLUSIONS}

Participants generally had limited knowledge about IVCM and the associated risk factors. Educational programs could be utilized to improve awareness and understanding.

\section{KEY WORDS}

Interventional Cardiology, Kidneys, Knowledge, Risk Factors, Contrast Media, Renal Function

\author{
Corresponding Author: \\ Dr. Rani Ghazi Ahmad, \\ Department of Radiology, \\ Faculty of Medicine, \\ King Abdulaziz University Hospital, \\ Jeddah, Saudi Arabia. \\ E-mail: rahmad@kau.edu.sa
}

DOI: $10.14260 /$ jemds/2020/534

How to Cite This Article:

Ahmad R, Almoallim R, Basalem D, et al. Awareness of intravenous contrast media effects on renal function in Saudi Arabiaan observational study. J Evolution Med Dent Sci 2020;9(34):2456-2460, DOI: 10.14260/jemds/2020/534

Submission 17-05-2020,

Peer Review 15-07-2020,

Acceptance 21-07-2020,

Published 24-08-2020.

Copyright (C) 2020 JEMDS. This is an open access article distributed under Creative Commons Attribution License [Attribution 4.0 International (CC BY 4.0)] 


\section{BACKGROUND}

Contrast media (CM), also termed contrast agents or contrast materials, enhance the images produced by various imaging techniques such as computed tomography (CT) and magnetic resonance imaging (MRI).[1] While CM is broadly considered to be safe, they can sometimes cause adverse reactions that should be evaluated before use.[2] Relatively uncommon moderate to severe reactions immediately following the use of CM include facial edema, bronchospasm, laryngeal edema, arrhythmias, bradycardia, tachycardia, hypertension or hypotension, pulmonary edema, and coronary artery spasm. ${ }^{[2]}$ Of the possible adverse effects caused by $\mathrm{CM}$, perhaps contrast-induced nephropathy (CIN) is among the most dangerous.[3] The European Society of Urogenital Radiology (ESUR) defines CIN as deterioration in renal function within 72 hours of intravenous administration of CM in the absence of other possible etiologies.[4] This deterioration is characterized by an elevation of serum creatinine to more than $25 \%$ from baseline levels or an increment of $0.5 \mathrm{mg} / \mathrm{dL}(44 \mu \mathrm{mol} / \mathrm{L}) .{ }^{[4]}$

Dayani et al.[5] has highlighted an association between contrast-induced acute kidney injury and poor clinical outcomes, such as short-term and long-term mortality, increased length of hospital stay, use of renal replacement therapy, and increased risk of cardiovascular disease.[6,7] Contrast-induced acute kidney injury is one of the most prominent types of acute renal damage, partly owing to the possibility of its prevention. The administration of intravenous contrast media (IVCM) enhances the CT scans through the differentiation of tissues (vascular and nonvascular), and enables accurate target volume and organ at risk delineation during radiotherapy planning. ${ }^{[8]}$

Currently, iodinated IVCM is required for accurate imaging, ${ }^{[3]}$ although it is the third most common cause of acute kidney injury, accounting for approximately $10-13 \%$ of cases. $[9,10]$ The risk could increase to $20 \%$ among patients with renal function impairments, while administration of $\mathrm{CM}$ accounts for a small number of renal injuries in the general population (0.6-2.3\%).[11,12] Pre-existing chronic kidney disease with concomitant diabetes mellitus is the highest risk factor associated with contrast-induced nephropathy. Other risk factors include advanced age, cardiovascular disease, and pre-procedure hemodynamic instability.[12-17] Zhang et al.[18] reported a negative impact of a high-dose of iodinated CM (iopamidol) among healthy rabbits, causing a marked decrease in renal function, detected by dynamic threedimensional magnetic resonance renography.

Contrast-induced nephropathy (CIN) is not only associated with longer hospitalization but also a higher risk of morbidity and mortality.[19] Public awareness of such consequences are not sufficient, which can put people at risk; indeed, ignorance of possible complications may lead to increased prevalence of the disease. A study conducted in Turkey revealed that $59.6 \%$ of patients thought that IVCM could be administered safely across various populations; most of the patients had inadequate knowledge of the possible serious complications of IVCM.[20] Indeed, among hospitalized patients, CIN has become an increasingly common cause of acute kidney injury. Al-Beladi et al.[21] showed a statistically insignificant association between patient age, gender, or body mass index and the development of CIN, but its prevalence was found to be higher in renal diseased groups even with similar demographic data.

Despite a worldwide yearly administration of 80 million IVCM doses, the decision concerning its clinical use for precipitating renal dysfunction remains multifaceted and complex.[22] As noted, the use of contrast media has been cited as one of the most common iatrogenic causes of acute kidney injury, and is linked with adverse outcomes; injury can begin with renal failure or dialysis initiation, and lead to stroke or even death. ${ }^{[23]}$ However, recent studies often show a broad consensus regarding the overall safety of IVCM usage for CT imaging, which do not show a significant risk to the general population for developing contrast-induced nephropathy or other potential adverse events in the six months following administration.[22]

Owing to the widespread use of IVCM in various fields of medicine, patient awareness and knowledge of CIN is particularly important, especially for high-risk groups. However, there is a paucity of studies that address public understanding of the association of CIN with longer hospitalizations and other adverse events. Therefore, the present study aims to assess the level of awareness of the risk of adverse events with IVCM, particularly with respect to renal function, surveying members of the public visiting a hospital in Saudi Arabia.

\section{METHODS}

\section{Study Design and Participants}

The study employed an observational cross-sectional design, recruiting 746 members of the general public visiting King Abdulaziz University Hospital (KAUH) in Jeddah, during September 2018.

\section{Inclusion and Exclusion Criteria}

All participants aged $\geq 18$ years recruited at KAUH were included in the study, although radiology staff and health workers that work with radiation were excluded.

\section{Patient and Public Involvement}

The participants were recruited in the hospital setting and informed about the study aims and procedure before the study commenced. The recruited participants were asked not to evaluate the risks and benefits of intervention.

\section{Ethical Considerations}

The study was approved by the Institutional Review Board (IRB) of KAUH, Jeddah, Saudi Arabia. All participants were required to sign a consent form.

\section{Data Collection}

Participants completed an electronic self-administered questionnaire to assess their knowledge of the possible adverse renal effects with IVCM. The questionnaire was validated by a panel of radiology experts. It consisted of 14 questions divided into two sections: the first section comprised questions on the demographic characteristics of 
the participants, such as age, sex, educational level, and occupation; the second section evaluated the knowledge of participants regarding the importance of IVCMs, its uses, possible renal adverse events, side effects, and risk factors. The second section also posed questions on the precautions and prophylactic measures associated with IVCM usage.

\section{Data Analysis}

The data were analyzed using the Statistical Package for the Social Sciences (SPSS), version 21. The analysis involved the use of descriptive and inferential statistics, comprising frequency tables and chi-squared tests to evaluate the level of knowledge of participants with respect to IVCM and their possible adverse renal effects. A P value of $<0.05$ was set as statistically significant.

\section{RESULTS}

The demographic characteristics of the participants are shown in Table 1. A total of 746 participants were incorporated into the study, among which, $67.4 \%$ were females and $32.6 \%$ were males. The predominant age group was $18-30$ years, which accounted for $44.8 \%$ of the cohort. More than half of the patients held a bachelor's degree (50.5\%).

Of the 746 participants included in the study, 129 (17.3\%) had been administered with IVCM for image enhancement. However, only $9.94 \%$ said that they were advised about possible side effects or adverse events from CM by health workers (Figure 1). Most of the participants (56.8\%) had no general knowledge of IVCM. Social media was the most chosen source of information for $32.3 \%$ of participants, which expressed variable degrees of awareness of IVCM. Furthermore, $51.9 \%$ of the participants that had general knowledge about IVCM did not know that it could have a significant impact on renal function, and also that it can cause further, more severe, adverse events, such as kidney failure and death. In those that claimed to have existing knowledge of IVCM, 48.1\% thought that the usual effect of IVCM was severe, and generally lead to renal failure. Concerning risk assessment of $\mathrm{CM}$ administration, $56.0 \%$ of the population that were aware of IVCM understood that anyone, regardless of age or renal condition, should undertake a renal function test when an IVCM enhanced analysis is required.

A total of $91.8 \%$ of participants thought that possible adverse effects should be discussed with the referring physician. However, most respondents lacked knowledge of the risk factors associated with CIN. Kidney disease was the most common risk factor recognized by participants, with $60.3 \%$ identifying it as a potential risk factor for developing CM-related complications (Figure 2). There was a statistically insignificant association between degree of knowledge, renal function test, and discussion of adverse effects with doctors with respect to gender (Table 2). Majority of the participants have no information of knowledge, and $55.7 \%$ male and $49 \%$ of female students thought that both pre and post-test are important with intravenous contrast administration. Both male and female students think that the possible adverse effects should be discussed with their doctor.

\begin{tabular}{|c|c|c|}
\hline \multicolumn{2}{|c|}{ Demographics } & Participants [N (\%)] \\
\hline \multirow{2}{*}{ Sex } & Male & $243(32.6)$ \\
\hline & Female & $503(67.4)$ \\
\hline \multirow{3}{*}{ Age } & $18-30$ & $334(44.8)$ \\
\hline & $31-40$ & $87(11.7)$ \\
\hline & $41-50$ & $143(19.2)$ \\
\hline \multirow{8}{*}{ Level of Education } & $51-60$ & $141(18.9)$ \\
\hline & 61 and above & $41(5.5)$ \\
\hline & Elementary & $3(0.4)$ \\
\hline & Intermediate & $19(2.5)$ \\
\hline & Secondary & $225(30.2)$ \\
\hline & Diploma & $83(11.1)$ \\
\hline & Bachelor & $377(50.5)$ \\
\hline & Higher education & $39(5.2)$ \\
\hline \multirow{2}{*}{ Health worker } & Yes & $78(10.5)$ \\
\hline & No & $668(89.5)$ \\
\hline
\end{tabular}

\begin{tabular}{|c|c|c|c|c|}
\hline & & \multicolumn{2}{|c|}{ Gender } & \multirow{2}{*}{ P-Value } \\
\hline & & Female & Male & \\
\hline \multirow{8}{*}{ Degree of knowledge } & Excellent & 13 & 10 & \multirow{8}{*}{0.129} \\
\hline & & $2.6 \%$ & $4.1 \%$ & \\
\hline & Good & 61 & 42 & \\
\hline & & $12.1 \%$ & $17.3 \%$ & \\
\hline & \multirow{2}{*}{ Not enough } & 139 & 57 & \\
\hline & & $27.6 \%$ & $23.5 \%$ & \\
\hline & information & $\begin{array}{r}290 \\
57.7 \%\end{array}$ & $\begin{array}{c}134 \\
55.1 \%\end{array}$ & \\
\hline & Important & 81 & 41 & \\
\hline \multirow{7}{*}{$\begin{array}{l}\text { When renal function } \\
\text { test should be done } \\
\text { with intravenous } \\
\text { contrast } \\
\text { administration? }\end{array}$} & pretest only & $16.1 \%$ & $16.9 \%$ & \multirow{7}{*}{0.410} \\
\hline & Important & 14 & 7 & \\
\hline & posttest only & $2.8 \%$ & $2.9 \%$ & \\
\hline & $\begin{array}{l}\text { Important pre } \\
\text { and post test }\end{array}$ & $\begin{array}{c}280 \\
55.7 \%\end{array}$ & $\begin{array}{c}119 \\
49.0 \%\end{array}$ & \\
\hline & Not & 7 & 6 & \\
\hline & $\begin{array}{l}\text { important } \\
\text { I don't }\end{array}$ & $\begin{array}{c}1.4 \% \\
121\end{array}$ & $\begin{array}{l}2.5 \% \\
70\end{array}$ & \\
\hline & $\begin{array}{l}1 \text { dont } \\
\text { know }\end{array}$ & $24.1 \%$ & $28.8 \%$ & \\
\hline \multirow{4}{*}{$\begin{array}{l}\text { Is it important to } \\
\text { discuss the possible } \\
\text { adverse effects with } \\
\text { your doctor? }\end{array}$} & Yes & 466 & 219 & \multirow{4}{*}{0.239} \\
\hline & res & $92.6 \%$ & $90.1 \%$ & \\
\hline & \multirow{2}{*}{ No } & 37 & 24 & \\
\hline & & $7.4 \%$ & $9.9 \%$ & \\
\hline Table 2. $\mathrm{Cr}$ & $\begin{array}{l}\text {-Tabulatio } \\
\text { and Diffe }\end{array}$ & $\begin{array}{l}\text { hi-Squar } \\
\text { t Variabl }\end{array}$ & ween $G$ & \\
\hline
\end{tabular}

\section{DISCUSSION}

This study has established that a large number of participants have indicated that they had no information regarding the possible adverse effects of IVCM administration. Indeed, most of the participants were not aware of the adverse effects of IVCM on renal function or their associated risk factors. This could be a product of the lack of sufficient healthcare programs to raise awareness among the general population, which could be exacerbated if there is a deficiency in the knowledge provided to patients by healthcare professionals during the decision-making process for IVCM administration. Similar results were found in a study conducted in Turkey in which $42.3 \%$ of the respondents had no information on the possible adverse effects of IVCM.[20]

Most of the participants with additional knowledge had a higher level of education or had prior exposure to IVCM. Similar findings have been found by Yucel et al.,[20] which showed a highly significant statistical association between the educational level of the participants and their knowledge about the effect and potential risk of IVCM on kidney function. However, this knowledge may be developed as a product of greater exposure to this imaging studies, so could be higher in this particular cohort than in the general population. The lack of knowledge among the general public, even in those with a high educational level, emphasizes the 
importance of increasing awareness about the potential renal risks of IVCM.[20] Adverse reactions are likely to be recognized early if individuals are aware of risk factors and are familiar with the importance of screening assessment before the administration of CM.[24] However, a similar finding of inadequate knowledge of predisposing factors to IVCM reactions was found in the cohort of the present study.

Based on the findings of this study, a number of significant implications for healthcare workers and radiologists dealing with IVCM and renal function have emerged. Participants lack awareness and understanding about appropriate patient screening and sufficient prophylactic measures, needed for the prevention of adverse reactions. While radiologists are highly familiar with the possible adverse renal effects of IVCM, strategies to lower their occurrence should be more widely introduced. This may include the initiation of programs that increase awareness among the general population and even healthcare workers that are not working with or directly utilizing radiological techniques.

It was found that most of the participants tended to obtain information about the imaging modality and possible adverse effects from social media. Therefore, healthcare providers should coordinate to improve public awareness through educational campaigns and programs, and possibly use the Internet and social media to bolster their impact.

The study may be limited because it has included only members of the general public that visited KAUH. This is probably not enough to build a definitive picture on the overall level of awareness of the general public across Saudi Arabia, although it may provide an estimate of public perception, since the hospital serves a large number of different communities with a variety of backgrounds. A further limitation of the study is its unequal representation of groups for gender, age, and education level. This was a product of the random distribution of the questionnaire and the demographics of the hospital population at the particular time of the survey. Considering these limitations, future research on this topic could be conducted in other major regions of the Middle East to gain a larger view of overall trends.

In 2001, the Radiological Society of Saudi Arabia (RSSA) has pledged to provide support for IVCM administration, and improve knowledge and training,[25] which should help remove delivery constraints for healthcare providers. While IVCM administration has been observed in developed countries, such as Ireland, USA, US, Europe, Canada, and Australia, similar studies have been limited in developing countries, which might have also had a negative impact on the level of knowledge and awareness.[25] Various regulatory frameworks could be introduced to enhance the administration of IVCM and integrate the role of the radiographer to address healthcare needs. If this study is replicated in different regions this may help attain national consensus about the limitations of current knowledge and awareness, and encourage a move towards a more widespread provision of information.

\section{CONCLUSIONS}

This study has highlighted the lack of awareness and information on the use of IVCM, and the inconsistency of understanding among participants. The heterogeneous background knowledge related to IVCM across various sites and the difference in educational level among participants are the major factors leading to insufficient understanding. A further factor hindering comprehension is the absence of definite guidelines concerning patient education on IVCM across various healthcare channels. While the findings of the present study concern the context of the general public of Saudi Arabia, the insights are relevant to IVCM practitioners in general, and support previous research about how patient understanding could be enriched and treatment improved in this area.[25] It is therefore recommended that routine clinical practice should include patient educational programs on IVCM so that more patients can be informed about the issues involved. More specific and widely available publications concerning the use of IVCM for patients at high risk should be developed in order to mitigate the issues arising in this group.

\section{List of Abbreviations}

- $\quad$ Computed Tomography (CT)

- Contrast Media (CM)

- Contrast-Induced Nephropathy (CIN)

- European Society of Urogenital Radiology (ESUR)

- Institutional Review Board (IRB)

- Intravenous Contrast Media (IVCM)

- $\quad$ King Abdullah University Hospital (KAUH)

- $\quad$ Statistical Package for the Social Sciences (SPSS)

\section{Declaration of Interest}

Ethical Approval- The study was approved by the Institutional Review Board (IRB) of KAUH, in Jeddah, Saudi Arabia.

Consent to Participate- All participants were required to sign a consent form before recruiting them into the study.

Consent for Publication- Not applicable.

Availability of Data and Materials- The datasets used and analyzed during the current study are available from the corresponding author upon request.

Competing Interest- The authors declare no competing interest.

\section{Acknowledgements}

The authors are thankful to all associated personnel who contributed to this research.

Financial or Other Competing Interests: None. 


\section{REFERENCES}

[1] Contrast materials. 2018. https://www.radiologyinfo.org/en/info.cfm?pg=safetycontrast

[2] Mruk B. Renal safety of iodinated contrast media depending on their osmolarity-current outlooks. Pol J Radiol 2016; 81:157-65.

[3] McCullough PA, Choi JP, Feghali GA, et al. Contrastinduced acute kidney injury. J Am Coll Cardiol 2016; 68(13):1465-73.

[4] European Society of Urogenital Radiology. 2018 http://www.esur.org/guidelines/

[5] Dayani MA, Mirzazadeh A. Prevention of contrastassociated acute kidney injury in cancer patients undergoing radiologic investigation using contrast media; a short-review to current knowledge. I Nephropathol 2019;8(4):e43.

[6] Bienholz A, Wilde B, Kribben A. From the nephrologist's point of view: diversity of causes and clinical features of acute kidney injury. Clin Kidney J 2015; 8(4):405-14.

[7] Launay-Vacher V, Janus N, Deray G. Renal insufficiency and cancer treatments. ESMO Open 2016;1(4):e000091.

[8] Minogue S, Gillham $C$, Kearney $M$, et al. Intravenous contrast media in radiation therapy planning computed tomography scans - current practice in Ireland. Tech Innov Patient Support Radiat Oncol 2019;12:3-15.

[9] Nash K, Hafeez A, Hou S. Hospital-acquired renal insufficiency. Am J Kidney Dis 2002;39(5):930-6.

[10] Stolker JM, McCullough PA, Rao S, et al. Pre-procedural glucose levels and the risk for contrast-induced acute kidney injury in patients undergoing coronary angiography. J Am Coll Cardiol 2010;55(14):1433-40.

[11] Mehran R, Nikolsky E. Contrast-induced nephropathy: definition, epidemiology, and patients at risk. Kidney Int Suppl 2006;100:S11-5.

[12] Manske CL, Sprafka JM, Strony JT, et al. Contrast nephropathy in azotemic diabetic patients undergoing coronary angiography. Am J Med 1990; 89(5):615-20.

[13] Kolonko A, Kokot F, Wiecek A. Contrast-associated nephropathy--old clinical problem and new therapeutic perspectives. Nephrol Dial Transplant 1998; 13(3):8036.
[14] Barrett BJ, Parfrey PS. Prevention of nephrotoxicity induced by radiocontrast agents. N Engl J Med 1994; 331(21):1449-50.

[15] Rudnick MR, Goldfarb S, Wexler L, et al. Nephrotoxicity of ionic and nonionic contrast media in 1196 patients: a randomized trial. The Iohexol Cooperative Study. Kidney Int 1995; 47(1):254-61.

[16] Aspelin P, Aubry P, Fransson SG, et al. Nephrotoxic effects in high-risk patients undergoing angiography. $\mathrm{N}$ Engl J Med 2003; 348(6):491-9.

[17] Jurado-Román A, Hernández-Hernández F, García-Tejada $\mathrm{J}$, et al. Role of hydration in contrast-induced nephropathy in patients who underwent primary percutaneous coronary intervention. Am J Cardiol 2015; 115(9):1174-8.

[18] Zhang YD, Wang J, Zhang J, et al. Effect of iodinated contrast media on renal function evaluated with dynamic three-dimensional MR renography. Radiology 2014; 270(2):409-15.

[19] Mohammed NMA, Mahfouz A, Achkar K, et al. Contrastinduced nephropathy. Heart Views 2013; 14(3):106-16.

[20] Yücel A, Değirmenci B, Acar M, et al. Patients' knowledge of the intravenous contrast materials and their risks: a cross-sectional survey. Turk J Med Sci 2005; 35:107-13.

[21] Al-Beladi FI. Cystatin C is an early marker of contrastinduced nephropathy in patients with sepsis in the intensive care unit. Saudi J Kidney Dis Transpl 2015; 26:718.

[22] Hinson JS, Ehmann MR, Fine DM, et al. Risk of acute kidney injury after intravenous contrast media administration. Annals of emergency medicine. 2017;69(5):577-86.

[23] Mitchell AM, Kline JA, Jones AE, et al. Major adverse events one year after acute kidney injury after contrastenhanced computed tomography. Annals of emergency medicine. 2015 Sep 1;66(3):267-74.

[24] Andreucci M, Solomon R, Tasanarong A. Side effects of radiographic contrast media: pathogenesis, risk factors, and prevention. Biomed Res Int 2014; 2014:1-20.

[25] Koch GGV, Swindon LD, Pillay JD. Medicolegal responsibilities for the administration of intravenous contrast media by radiographers: radiologists' perspectives. South Afr J Bioethics Law 2018;11(2):60-5. 